\title{
Translation Between Two Models; Application with Integrated Glucose Homeostasis Models
}

\author{
Moustafa M. A. Ibrahim ',2 • Anna Largajolli ' Maria C. Kjellsson ' • Mats O. Karlsson '
}

Received: 4 September 2018 / Accepted: 18 February 2019 / Published online: 17 April 2019

(C) The Author(s) 2019

\begin{abstract}
Purpose For some biological systems, there exist several models with somewhat different features and perspectives. We propose an evaluation method for NLME models by analyzing real and simulated data from the model of main interest using a structurally different, but similar, NLME model. We showcase this method using the Integrated Glucose Insulin (IGI) model and the Integrated Minimal Model (IMM). Additionally, we try to map parameters carrying similar information between the two models.

Methods A bootstrap of real data and simulated datasets from both the IMM and IGI models were analyzed with the two models. Important parameters of the IMM were mapped to IGI parameters using a large IMM simulated dataset analyzed under the IGI model.

Results Comparison of the parameters estimated from real data and data simulated with the IMM and analyzed with the IGI model demonstrated differences between real and IMM-simulated data. Comparison of the parameters estimated from real data and data simulated with the IGI model and analyzed with the IMM also demonstrated differences but to a lower extent. The strongest parameter correlations were found for: insulin-dependent glucose clearance (IGI) insulin sensitivity (IMM); insulin-independent glucose clearance (IGI) $\sim$ glucose effectiveness (IMM); and insulin effect parameter (IGI) $\sim$ insulin action $(\mathrm{IMM})$.
\end{abstract}

Electronic supplementary material The online version of this article (https://doi.org// 0. I007/s I 1095-019-2592-9) contains supplementary material, which is available to authorized users.

Mats O. Karlsson

mats.karlsson@farmbio.uu.se

Department of Pharmaceutical Biosciences, Uppsala University Box 59I, 75I 23 Uppsala, Sweden

2 Department of Pharmacy Practice, Helwan University Cairo, Egypt
Conclusions We demonstrated a new approach to investigate models' ability to simulate real-life-like data, and the information captured in each model in comparison to real data, and the IMM clinically used parameters were successfully mapped to their corresponding IGI parameters.

KEY WORDS glucose effectiveness · insulin sensitivity . integrated glucose insulin model · integrated minimal model · NLMEPC

\section{INTRODUCTION}

Nonlinear mixed effects (NLME) modeling is increasingly used to describe longitudinal data from clinical experiments to increase our understanding of the studied underlying pathophysiological system (1). NLME models allow the pooling of data from different subjects while simultaneously quantifying multiple levels of variability. This ability to identify different sources of variability is particularly critical to many clinical applications and has motivated the implementation of previously developed milestone models of biological systems into the NLME context. For some biological systems, there exist several models with somewhat different features. This may have arisen as the underlying data may have been different allowing a more or less detailed description of the system, the developers of the models may have had different insights or the purpose of the models may have been different. The resulting diversity provides detailed insights into the studied biological system from different perspectives, with learning opportunities from the comparison across models. Here, we present a comparison between two models in the field of diabetes.

In diabetes, Ajmera et al. (2) presented a detailed review of the modeling efforts undertaken to describe glucose homeostasis, diabetes, and related complications. Among the numerous models developed to describe glucose-insulin regulation 
following system perturbation, the minimal models are regarded as the first breakthrough in diabetes modeling. (3-7) They are not data-driven, rather parsimoniously formulated based on physiology. Glucose minimal model provides two important clinical indices that are able to describe the system in different pathophysiological states, glucose effectiveness index $\left(\mathrm{S}_{\mathrm{G}}\right)$ that quantifies the ability of glucose to enhance its own rate of disappearance at basal insulin concentration and insulin sensitivity index $\left(\mathrm{S}_{\mathrm{I}}\right)$ that quantifies the influence of insulin at steady state to change glucose's own effect on glucose concentration. To overcome several limitations, glucose minimal model went through many developments driven by its validation versus clinical studies and was adapted to NLME modeling (8). However, these models are conditional models that describe either glucose or insulin and use the other variable as a known input. This feature limited their simulation performance and utilization for clinical drug development. Thus, integrated models that can describe simultaneously glucose and insulin kinetics following system perturbation were in need. Though with different structure model assumptions, the integrated glucose insulin (IGI) model (9) then the integrated minimal model (IMM) (10) have been developed by NLME modeling to fill this gap and describe glucose-insulin regulation system following, for example, intravenous glucose tolerance test (IVGTT).

A major step in the evaluation of NLME models was when the properties of multiple data sets simulated from the model with the original trial design was compared to the original, real data using standard metrics in posterior predictive checks (PPCs) (11-13) or graphically in visual predictive checks (VPCs) (14-16). The common principle in these evaluations are that any systematic discrepancy between the observed data metric and the distribution given by the multiple simulated data sets would indicate a model deficiency. However, the PPC typically focuses on one or two metrics of clinical interest and the VPC evaluate the match in distribution of data at different times following the start of study but does not provide an evaluation of the relative contribution of the different components creating variability between observations. Also, neither technique is well-developed for multivariable models, like the glucose-insulin models.

In this paper, we propose a new evaluation form of simulated data and that is the analysis of both real and simulated data using a structurally different but similar NLME model and hence we called it "NLMEPC" in analogy with VPC and PPC. As NLME models assess both the expected time-courses and inter- and intra-individual variability as well as interdependence between dependent variables, it will pose a challenging test for the simulated data. We demonstrate NLMEPC using two similar NLME models: the IGI model and the IMM. Both models had been proposed to describe the same biological system with different structural assumptions, and this would allow performing such evaluation crosswise, i.e. the IGI model can be used to assess the IMM and vice versa. Additionally, we present an approach to map parameters carrying similar information between NLME models. We showcase this approach by trying to map the clinically used parameters available in the IMM with their corresponding parameters in the IGI model.

\section{METHODS}

\section{NLME Models}

The IGI model shown in Fig. 1, was developed for both healthy subjects and patients with type 2 diabetes mellitus (T2DM) following labeled IVGTT, using observations of glucose, labeled glucose (tracer) and insulin. Glucose sub-model is a two-compartment model, $\mathrm{G}_{\mathrm{C}}(\mathrm{mg})$ and $\mathrm{G}_{\mathrm{P}}(\mathrm{mg})$ are glucose amounts in central and peripheral compartments with their volumes of distributions $\mathrm{V}_{\mathrm{G}}(\mathrm{L})$ and $\mathrm{V}_{\mathrm{P}}(\mathrm{L})$ respectively, and inter-compartmental clearance $\mathrm{Q}\left(\mathrm{L} \cdot \mathrm{min}^{-1}\right)$. Elimination from the central compartment is divided into insulin-dependent clearance $\mathrm{CL}_{\mathrm{GI}}\left(\mathrm{L} \cdot \mathrm{min}^{-1} / \mathrm{mU} . \mathrm{L}^{-1}\right)$ and insulin-independent clearance $\mathrm{CL}_{\mathrm{G}}\left(\mathrm{L} \cdot \mathrm{min}^{-1}\right)$. Glucose sub-model has two effect compartments, $\mathrm{G}_{\mathrm{E} 1}(\mathrm{mg} / \mathrm{dL})$ and $\mathrm{G}_{\mathrm{E} 2}(\mathrm{mg} / \mathrm{dL})$ are glucose concentrations in these effect compartments accounting for the control mechanisms of glucose on its own production and on the 2nd phase insulin secretion respectively, $\mathrm{k}_{\mathrm{GE} 1}\left(\mathrm{~min}^{-1}\right)$ and $\mathrm{k}_{\mathrm{GE} 2}\left(\mathrm{~min}^{-1}\right)$ are their respective rate constants while GPRG and IPRG are power terms mediating these control mechanisms respectively. The labeled glucose sub-model is assumed to have the same characteristics as the glucose submodel but without hepatic glucose production. Insulin sub-

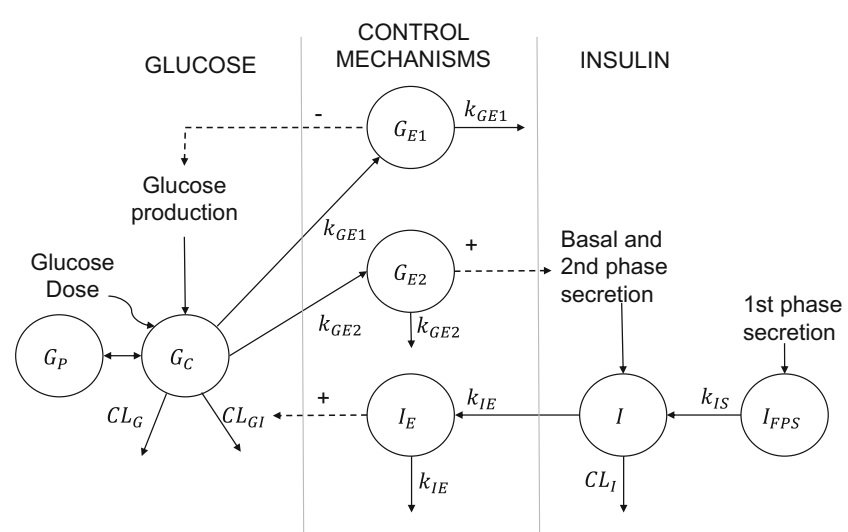

Fig. I Schematic presentation of the integrated glucose insulin model during IVGTT in healthy subjects. $G_{C}$ and $G_{p}$, central and peripheral compartments of glucose; $G_{E 1}$ and $G_{E 2}$, effect compartments of glucose on endogenous glucose production and 2nd phase insulin secretion respectively; I, central compartment of insulin; $I_{E}$, effect compartment of insulin on glucose; $I_{F P S}$, insulin Ist phase compartment; $k_{G E I}, k_{G E 2}, k_{I E}$ and $k_{I S}$, first order rate parameters; $\mathrm{CL}_{\mathrm{G}}, \mathrm{CL}_{\mathrm{Gl}}$ and $\mathrm{CL}_{1}$, insulin-independent glucose clearance, insulin-dependent glucose clearance and insulin clearance respectively. 
model is a one compartment disposition model, with the volume of distribution $\mathrm{V}_{\mathrm{I}}(\mathrm{L})$ and the clearance $\mathrm{CL}_{\mathrm{I}}\left(\mathrm{L} \cdot \mathrm{min}^{-1}\right)$. Insulin sub-model has one effect compartment for the delayed effect of insulin on the regulation of $\mathrm{CL}_{\mathrm{GI}}$ with $\mathrm{k}_{\mathrm{IE}}\left(\mathrm{min}^{-1}\right)$ as the rate constant. Upon glucose administration, insulin 1st phase amount IFST (mU) enters insulin 1st phase compartment as a system's response, then it is released into insulin central compartment through the rate constant $\mathrm{k}_{\mathrm{IS}}\left(\mathrm{min}^{-1}\right)$. $\mathrm{G}_{\mathrm{SS}}\left(\mathrm{mg} . \mathrm{L}^{-1}\right)$ and $\mathrm{I}_{\mathrm{SS}}\left(\mathrm{mU}_{\mathrm{L}} \mathrm{L}^{-1}\right)$ are the basal concentrations of glucose and insulin respectively. The IGI model was the first proposed integrated semi-mechanistic model to describe both glucose and insulin data simultaneously. One of the appealing features of the IGI model is its ability to distinguish hepatic glucose production from glucose disappearance, facilitated by access to labelled glucose measurements during its development. The model had been successfully applied for exploring drug effects (17), disease progression (18), designing early clinical trials (19) and optimizing IVGTT design (20).

The IMM was recently proposed to overcome the limitations of the traditional minimal models, while still deriving the important physiological indices for clinical diagnosis, $\mathrm{S}_{\mathrm{G}}$ and $\mathrm{S}_{\mathrm{I}}$. The model was developed in healthy subjects following an insulin modified IVGTT, but its data was lacking the unique information provided by labeled glucose. The model is divided into two sub-models, glucose and insulin, based on the twocompartment glucose minimal model (5) and insulin minimal model (7) respectively, as shown in Fig. 2. Glucose sub-model is a two-compartment model, and $\mathrm{G}_{1}(\mathrm{mg})$ and $\mathrm{G}_{2}(\mathrm{mg})$ are glucose amounts in the central and the peripheral compartments with their volumes of distributions VOL $(\mathrm{dL})$ and VOLI (dL) respectively. Elimination is from the central compartment only. Transit compartments are used to cater for glucose kinetics in the first minutes after glucose dosing. The rate of change of glucose amount in the central compartment is the difference between the rate of hepatic glucose production $\mathrm{Ra}\left(\mathrm{mg} \cdot \mathrm{min}^{-1}\right)$, the rate of glucose disappearance by liver uptake $\mathrm{Rdl}$ (mg.min ${ }^{-1}$ ), the rate of glucose disappearance by peripheral tissue $\operatorname{Rdp}\left(\mathrm{mg} \cdot \mathrm{min}^{-1}\right)$, and the distribution between the central and the peripheral compartments. Since unlabeled IVGTT data did not allow the explicit description of hepatic glucose production, both hepatic glucose production and glucose liver uptake were lumped together as the net hepatic glucose balance (.NHGB). Insulin sub-model consists also of a twocompartment model, VI (L) and VI2 (L) are the volumes of distributions of the central and the peripheral compartments respectively. Elimination from the central compartment is described by the clearance enneCL (L.min ${ }^{-1}$ ) through the rate constant $\mathrm{k}_{\mathrm{I}}\left(\mathrm{min}^{-1}\right)$. Transit compartments with the mean transit time MTT3 ( $\mathrm{min}$ ) are used to account for the delay in insulin 1st phase amount x0 (pmol.L $\left.{ }^{-1}\right)$. While 2nd phase insulin secretion rate $\mathrm{Y}$ (pmol. $\mathrm{Min}^{-1}$ ) is proportional to glucose concentration. ALPH $\left(\mathrm{min}^{-1}\right)$ is a time constant parameter that controls the decrease in $\mathrm{Y}(\mathrm{t})$ toward a steady state value, BETA (pmol.min ${ }^{-1} . \mathrm{L}^{-1} / \mathrm{mg} . \mathrm{dL} \mathrm{L}^{-1}$ ) is the feedback control of glucose concentration in glucose central compartment above basal glucose concentration $\mathrm{H}\left(\mathrm{mg} \cdot \mathrm{dl}^{-1}\right)$ on $\mathrm{Y}$. When insulin

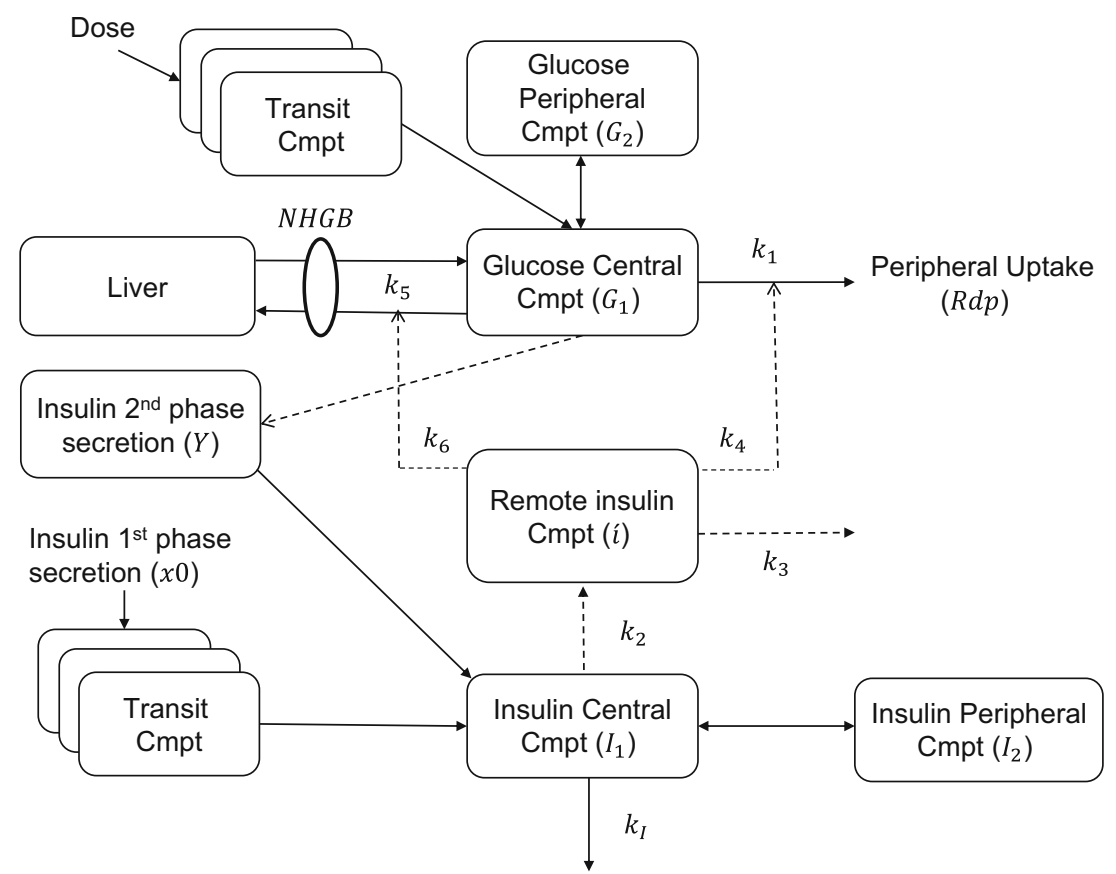

Fig. 2 Schematic presentation of the integrated minimal model during IVGTT in healthy subjects. G, and $G_{2}$, central and peripheral compartments of glucose; I and $\mathrm{I}_{2}$, central and peripheral compartments of insulin; $\boldsymbol{l}$ insulin concentrations in the remote compartment; Rdp, rate of glucose disappearance by peripheral tissue uptake; $x 0$, I st phase insulin concentrations; $Y, 2$ 2nd phase insulin secretion; NHGB, net hepatic glucose balance; $k_{1}$ and $k_{5}$, glucose model parameters; $k_{2}$, $k_{3}, k_{4}$ and $k_{6}$ parameters of insulin action; $k_{1}$, insulin elimination rate constant. 
concentrations in the central compartment are higher than its basal concentrations QUINS (pmol.L ${ }^{-1}$ ), it moves to a remote compartment representing the receptor pool for insulin binding to peripheral tissues, where it produces its effect by increasing glucose disappearance. QE1 (L.min $\left.{ }^{-1}\right)$ and QE2 (L.min $\left.{ }^{-1}\right)$ are the inter-compartmental clearances describing the distribution of glucose and insulin respectively. $\mathrm{k}_{1}\left(\mathrm{~min}^{-1}\right)$ and $\mathrm{k}_{4}$ (L. pmol ${ }^{-1}$. min $^{-1}$ ) are rate parameters that describe peripheral uptake as a function of glucose amount in the central compartment and insulin concentration in the remote compartment respectively. $\mathrm{k}_{2}\left(\mathrm{~min}^{-1}\right)$ and $\mathrm{k}_{3}\left(\mathrm{~min}^{-1}\right)$ are rate parameters for insulin effect, $\mathrm{k}_{5}\left(\mathrm{~min}^{-1}\right)$ is a rate parameter that describes hepatic glucose uptake as well as the inhibitory effect of glucose on hepatic glucose production, $\mathrm{k}_{6}\left(\mathrm{~L} \cdot \mathrm{pmol}^{-1} \cdot \mathrm{min}^{-1}\right)$ is a rate parameter that describes the effect of insulin concentration in the remote compartment on glucose to enhance hepatic glucose uptake, as well as the inhibitory effect of insulin on hepatic glucose production. For a priori identifiability of model parameters, the model was reparametrized as:

$$
\begin{aligned}
p 1 & =\mathrm{k}_{1}+\mathrm{k}_{5} \\
p 2 & =\mathrm{k}_{3} \\
p 3 & =\mathrm{k}_{2} \cdot\left(\mathrm{k}_{4}+\mathrm{k}_{6}\right) \\
\mathrm{SI} & =\frac{p 3}{p 2} \\
\mathrm{~S}_{\mathrm{G}} & =p 1 \cdot \mathrm{VOL} \\
\mathrm{S}_{\mathrm{I}} & =\mathrm{SI} \cdot \mathrm{VOL}
\end{aligned}
$$

While $\mathrm{S}_{\mathrm{G}}$ is an estimated model parameter, $\mathrm{S}_{\mathrm{I}}$ need to be calculated separately after model estimation from SI and VOL. This integrated model provides estimates of $\mathrm{S}_{\mathrm{G}}$ and $\mathrm{S}_{\mathrm{I}}$ that are compatible with the traditional minimal model approaches while allowing the simultaneous characterization of the glucose-insulin regulation system (10). The difference between $\mathrm{S}_{\mathrm{G}}$ and $\mathrm{S}_{\mathrm{I}}$ derived by the IMM and those derived by the original minimal model (5) is the use of insulin predictions to drive parameters' estimates in the IMM, while the original minimal model uses insulin observations. This difference will be minimal when the IMM describes insulin observations adequately. More details on the IMM system of differential equations can be found in the Appendix where we maintained the original minimal models' notations.

\section{Data}

Real data was used from two previously published studies, the first study was a stable isotopically labeled IVGTT in 14 healthy volunteers, where an intravenous bolus dose of glucose, enriched with labeled glucose, was administered, and frequent blood samples were drawn for $240 \mathrm{~min}$ following the glucose dose (21). The second study was an insulinmodified stable isotopically labeled IVGTT in 10 healthy volunteers. An intravenous bolus dose of glucose enriched with labeled glucose was administered, and after $20 \mathrm{~min}$, a 5-min insulin infusion was given. Frequent blood samples were drawn for 240 min following the glucose dose (22). In both studies, plasma samples were analyzed to determine the concentration of total glucose, insulin, and labeled glucose. We did not use the labeled glucose data since the IMM was developed by unlabeled IVGTT data. The two models were fitted to the real data to obtain the point estimates that were used later for the simulations and as the initial guess for estimations. Visual predictive checks (VPCs) were performed to investigate the adequacy of each model initial fit.

\section{NLMEPC Procedures}

Three hundred data sets were created from three different data sources, one hundred data sets were sampled from the real data by bootstrap, one hundred data sets were simulated from the point estimates of the IMM using the same study design as the real data, and lastly one hundred data sets were simulated from the point estimates of the IGI model using also the same study design as the real data. Then the two models were fitted to each of the created three hundred datasets as shown in Fig. 3. This resulted in three hundred estimates for each model parameter, these are rich enough to investigate each of the parameters distribution properties and provide additional insights to where the model failed through understanding the differences in parameters distributions across the three different data sources, as well as investigating the information content of each model's simulated data compared to real-life-like data (bootstrap datasets).

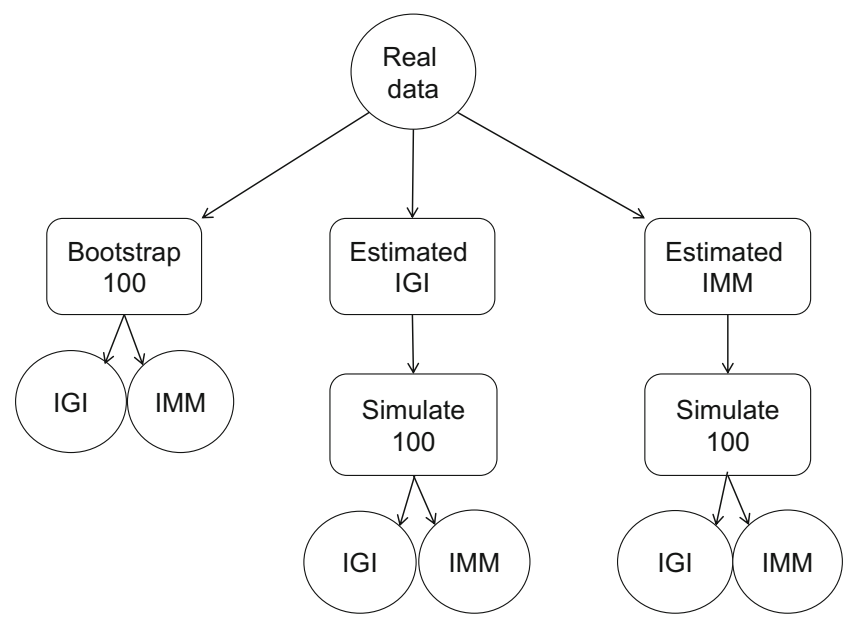

Fig. 3 A descriptive presentation of information translatability investigation set up. The two models were first fitted to the real data. Afterward, one hundred data sets were sampled from the real data by bootstrap, one hundred datasets were simulated from the IMM, and lastly one hundred datasets were simulated the IGI model. Then the two model were fitted to each of the created three hundred datasets. 


\section{Mapping the Clinical Parameters}

For mapping glucose effectiveness $\mathrm{S}_{\mathrm{G}}$, insulin sensitivity $\mathrm{SI}$ and insulin action $p 2$ derived by the IMM with their expected corresponding parameters in the IGI model $\left(\mathrm{CL}_{\mathrm{G}}, \mathrm{CL}_{\mathrm{GI}}\right.$ and $\left.\mathrm{k}_{\mathrm{IE}}\right)$, a single large dataset (1200 individuals) was simulated from the IMM. The simulated individual estimates of all IMM parameters were outputted in this large dataset. This was followed by a single estimation step by the IGI model, and the individual estimates of $\mathrm{CL}_{\mathrm{G}}, \mathrm{CL}_{\mathrm{GI}}$, and $\mathrm{k}_{\mathrm{IE}}$ were outputted as well. Then the simulated individual estimates of all IMM parameters from the simulation step were treated as true covariates and added on the individual parameter estimates of the $\mathrm{CL}_{\mathrm{G}}, \mathrm{CL}_{\mathrm{GI}}$, and $\mathrm{k}_{\mathrm{IE}}$ from the estimation step (treated as response variables) using generalized additive modeling GAM approach (23) as shown in Fig. 4. GAM is an extension of generalized linear models. Generalized linear models relates the expected value of the response variable $(\mu)$ to a linear function of the covariates $(X \mathrm{~s})$ multiplied by their respective coefficients $(\beta)$ :

$\mu=\beta_{0}+\beta_{1} \cdot X_{1}+\beta_{2} \cdot X_{2}+\beta_{3} \cdot X_{3}$

The generalization here is the ability to drive such relations for response variables that are not normally distributed, through transforming these response variables by link function $g($.$) , e.g. natural logarithm can be used as a link function for a$ response variable of a Poisson distribution with mean and variance $\mu$ :

$$
g(\mu)=\beta_{0}+\beta_{1} \cdot X_{1}+\beta_{2} \cdot X_{2}+\beta_{3} \cdot X_{3}
$$$$
\ln (\mu)=\beta_{0}+\beta_{1} \cdot X_{1}+\beta_{2} \cdot X_{2}+\beta_{3} \cdot X_{3}
$$

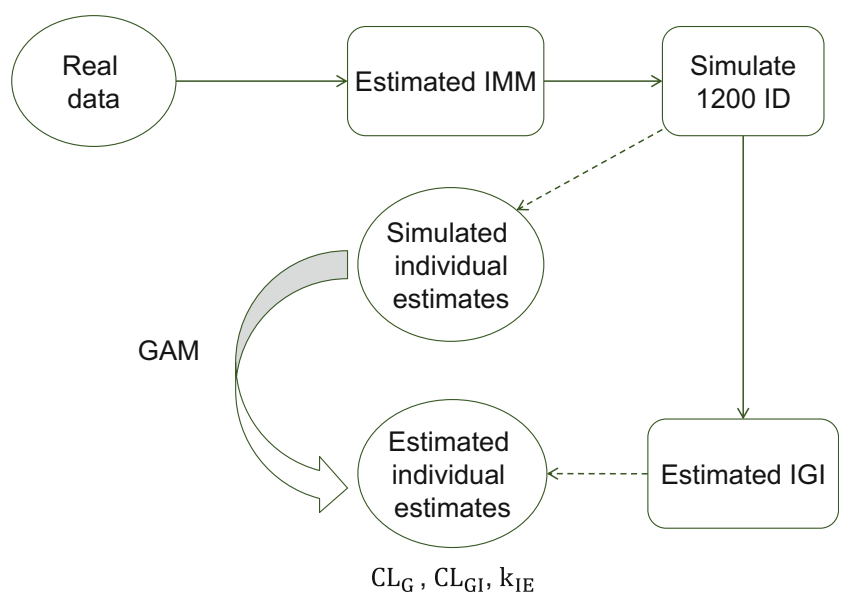

Fig. 4 A descriptive representation of the setup for mapping physiological indices derived from the IMM to their corresponding parameters in the IGI model. A large data set ( 1200 Individuals) was simulated from the IMM, then estimated by the IGI model. Thereafter the simulated individual estimates of all IMM parameters were treated as true covariates and added on the estimated individual estimates of the $\mathrm{CL}_{\mathrm{G}}, \mathrm{CL}_{\mathrm{Gl}}$, and $\mathrm{K}_{\mathrm{IE}}$ using $\mathrm{GAM}$ approach. $\mathrm{CL}_{\mathrm{G}}$, insulin-independent glucose clearance; $\mathrm{CL}_{\mathrm{Gl}}$, insulin-dependent glucose clearance; $K_{\mathrm{IE}}$, insulin effect rate constant.
An extra layer of generalization is then obtained by GAM that allows the testing of nonlinear functions $f($.$) of$ the covariates to establish their relationship with the response variable:

$\mu=\beta_{0}+f\left(X_{1}\right)+f\left(X_{2}\right)+f\left(X_{3}\right)$

Thus, generalized additive models are more flexible with better fits to the data than generalized linear models. For each response variable $\left(\mathrm{CL}_{\mathrm{G}}, \mathrm{CL}_{\mathrm{GI}}\right.$, and $\left.\mathrm{k}_{\mathrm{IE}}\right)$, we used stepwise GAM for selection among the different parameters of the IMM using Akaike information criterion (AIC). This means that for each response variable, all the IMM parameters $\left(\mathrm{IMM}_{\text {parameter }}\right)$ were fitted separately in the GAM (one fit for each $\mathrm{IMM}_{\text {parameter }}$ ) and AIC was calculated for each fit. The $\mathrm{IMM}_{\text {parameter }}$ that was accompanied by the highest improvement in fit was added first in the GAM, then the rest of parameters were tested again on the new generalized additive model (that included the first $\mathrm{IMM}_{\text {parameter }}$ ) to identify the $\mathrm{IMM}_{\text {parameter with the highest improvement of fit in the new }}$ generalized additive model that would be the second $\mathrm{IMM}_{\text {parameter }}$ added by the GAM and so on. Afterwards, the first $\mathrm{IMM}_{\text {parameter }}$ to be added by the GAM, was incorporated as a covariate on the population parameter of $\mathrm{CL}_{\mathrm{G}}, \mathrm{CL}_{\mathrm{GI}}$, and $\mathrm{k}_{\mathrm{IE}}$ in a second re-estimation step by the IGI model for the same simulated large dataset as shown in (Eq. 7) using $\mathrm{CL}_{\mathrm{G}}$ as an example, where $\theta_{\mathrm{CL}_{\mathrm{G}}}$ is the population parameter for $\mathrm{CL}_{\mathrm{G}}$, $\theta_{\mathrm{EFFECT}}$ is a measure of change in $\mathrm{CL}_{\mathrm{G}}$ for each unit change in the added $\mathrm{IMM}_{\text {parameter, }}$ and $\eta_{\mathrm{CL}_{\mathrm{G}}}$ is the unexplained individual deviation from the population parameter $\theta_{\mathrm{CL}_{\mathrm{G}}}$. This explored how much of the inter-individual variability in each of these parameters of the IGI model was explained by incorporating these covariates. Nonlinear mixed effects analysis, statistical and graphical assessment was performed in PSN, (24) NONMEM version 7.3 (25) and R (26).

$$
\mathrm{CL}_{\mathrm{G}}=\left(\theta_{\mathrm{CL}_{\mathrm{G}}}+\theta_{\mathrm{EFFECT}} * \mathrm{IMM}_{\text {parameter }}\right) * \exp ^{\eta_{\mathrm{CL}_{\mathrm{G}}}}
$$

\section{RESULTS \\ NLMEPC}

As expected, both models described the data well as shown by their respective VPCs in Fig. 5, providing the evidence for adequate parameters' estimates from the initial fit. The IMM described insulin observations adequately at the individual level as shown by a goodness of fit plot of insulin observations versus insulin predictions (Supplementary material). Plots of the 300 estimates of each parameter of the IGI model, grouped by the three different sources of the data are shown in Fig. 6. The simulation performance of the two models can be judged by comparing to the bootstrap data which is 

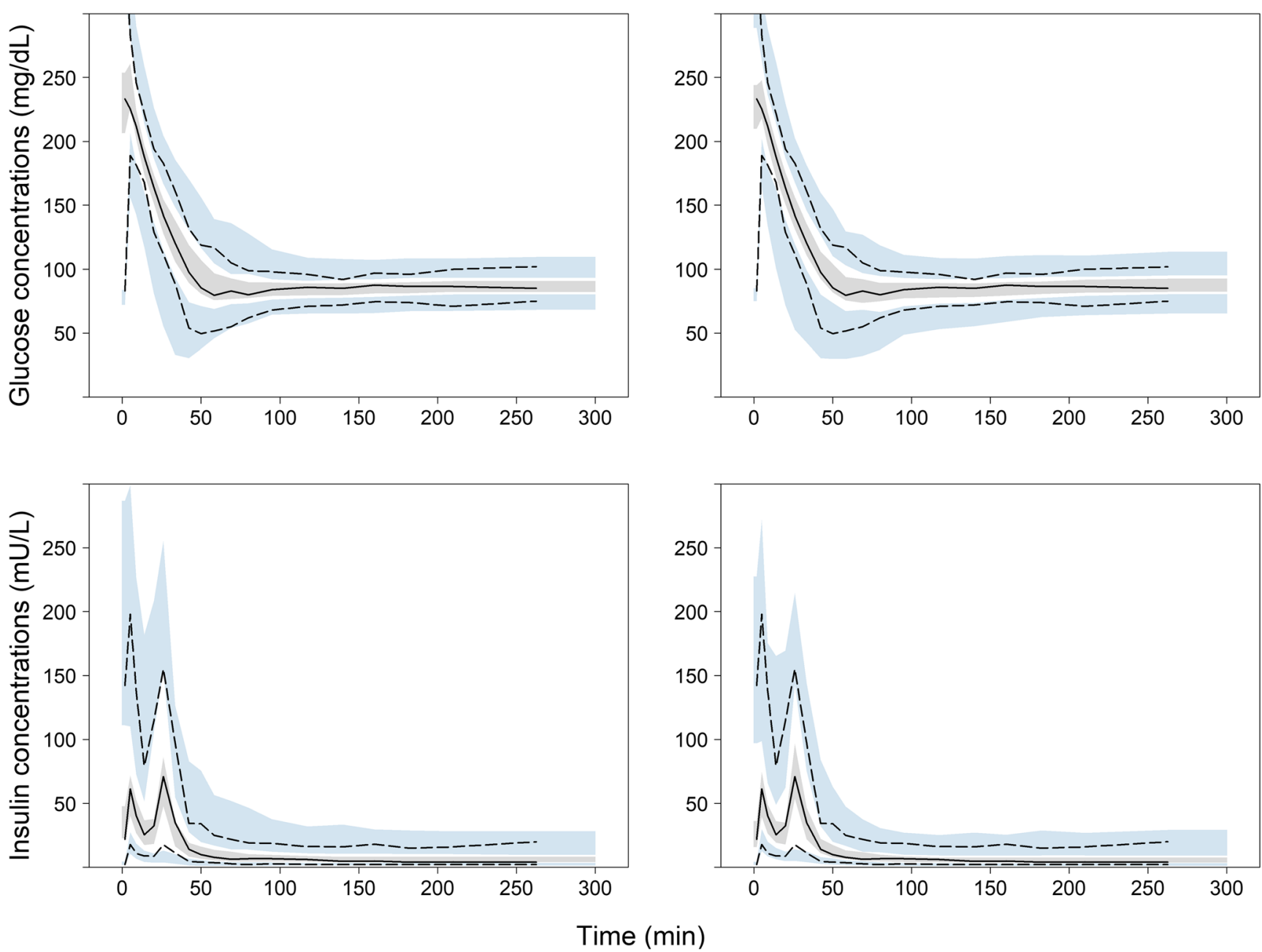

Fig. 5 Visual predictive checks of the two dependent variables (glucose and insulin) from each model initial fit. The IGI model on the right-hand side and the IMM on the left-hand side, the lines represent the median of the data, the dashed lines are the 5th, 95th percentiles of the data. The shaded areas are the confidence intervals around the same percentiles in the simulated data. Both models described the data well as the median, 5th, 95th percentiles of the data are within the shaded areas.

assumed to represent the truth or real-life data, i.e. looking at $\mathrm{G}_{\mathrm{SS}}$ or $\mathrm{k}_{\mathrm{GE} 2}$ parameter plots, both models' simulations contained enough information to estimate these parameters with a similar distribution to bootstrap data. There was no difference in any of parameters distribution between the IGI model simulations and bootstrap data even though the medians did not overlap in some cases, however, $75 \%$ spread of the IMM simulations was below or above $75 \%$ spread of bootstrap in $\mathrm{CL}_{\mathrm{G}}, \mathrm{CL}_{\mathrm{GI}}, \mathrm{GPRG}, \mathrm{IPRG}, \mathrm{I}_{\mathrm{SS}}, \mathrm{k}_{\mathrm{IE}}, \mathrm{k}_{\mathrm{IS}}$, Q $V_{G}$, and $V_{I}$ parameters. The estimated distribution of GPRG from the data simulated by the IMM was shifted toward zero. That concluded an obvious difference in the information content of the IMM simulations and bootstrap for these parameters. Additionally, simulations from the IMM showed large uncertainty with the remaining two parameters $\mathrm{k}_{\mathrm{GE} 1}$ and $\mathrm{V}_{\mathrm{P}}$ where $\mathrm{k}_{\mathrm{GE} 1}$ estimates were also close to zero. In fact, GPRG and $\mathrm{k}_{\mathrm{GE} 1}$ estimated distributions from the IMM are conveying a message about the implicit description of endogenous glucose production in the IMM. In general, the precision was good for bootstrap and when the same model is used for simulation and estimation, i.e. the IGI model, while the lowest precision is seen with the IMM simulations.

Similarly, plots of the 300 estimates of each parameter of the IMM, grouped by the three different sources of the data are shown in Fig. 7. Simulations from both models contained enough information to estimate $\mathrm{S}_{\mathrm{G}}$, VOL, SI, QE1, enneCL, BETA, $\mathrm{H}$, and QUINS parameters with a similar distribution as bootstrap data. Surprisingly, the 50\% spread of the IMM simulations were above $75 \%$ spread of bootstrap data for parameters $p 2$ and VI, so there is likely different information content between these two data sources. The same problem was found for parameter ALPH, $p 2$ and $\mathrm{x} 0$ with the IGI model simulations whose $75 \%$ spread was below $50 \%$ spread of bootstrap and thus their information content is likely to be different, however for parameter VI, there was no difference between these two data sources. The $75 \%$ spread of the IGI model simulations were below $75 \%$ spread of bootstrap for parameters VOLI, QE2 and MTT3 and so there is an 
IGI parameters
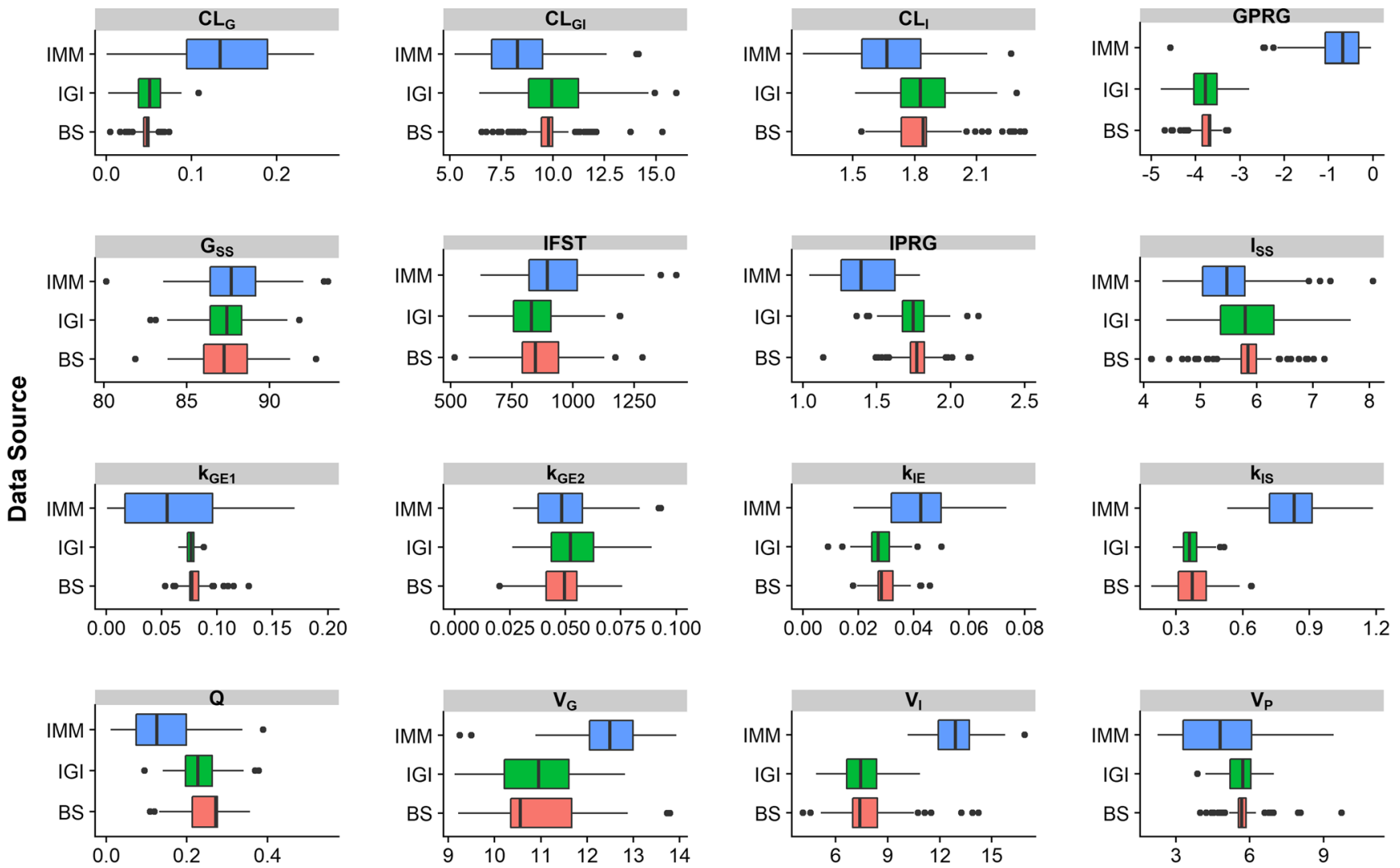

\section{Estimates}

Fig. 6 Boxplots of the estimated distributions of each parameter of the IGI model, grouped by the three different sources of the data: one hundred bootstrap datasets (red), one hundred datasets simulated from the IGI model (green) and one hundred datasets simulated from IMM model (blue). The boxplot length is the interquartile range (the middle half of the distribution) while each whisker represents one quarter of the distribution. BS, bootstrap; IGI, integrated glucose insulin model; IMM, integrated minimal model; $\mathrm{CL}_{\mathrm{G}}, \mathrm{CL}_{\mathrm{Gl}}$ and $\mathrm{CL}_{1}$, insulin-independent glucose clearance, insulin-dependent glucose clearance and insulin clearance; GPRG, power parameter for glucose effect on endogenous glucose production; $\mathrm{G}_{\mathrm{ss}}$, glucose basal concentrations; IFST, I st phase insulin amount; IPRG, power parameter for glucose effect on 2 nd phase insulin secretion; $I_{5 s}$, insulin basal concentrations; $k_{G E I}, k_{G E 2}, k_{I E}$ and $k_{I S}$, first order rate parameters; $Q$, glucose intercompartmental clearance; $V_{G}, V_{1}, V_{P}$, volumes of distribution of glucose and insulin central compartments, glucose peripheral compartment.

obvious difference in the information content of these two data sources, but no difference can be claimed between the IMM simulations and the bootstrap data for these three parameters. Interestingly, parameter VI2 was well estimated from the IGI model simulations even though this model has only one compartment disposition model for insulin kinetics. Finally, the precision for all parameters was good with the three different sources, with the highest precision with the IMM as expected.

\section{Mapping the Clinical Parameters}

Each of the individual simulation parameter values for $\mathrm{S}_{\mathrm{G}}$, SI and $p 2$ was the first parameter to be added by the GAM as a true covariate on $\mathrm{CL}_{\mathrm{G}}, \mathrm{CL}_{\mathrm{GI}}$, and $\mathrm{k}_{\mathrm{IE}}$ individual estimates, respectively. These were the strongest correlations of the physiological indices and insulin action with the IGI model parameters. On incorporating these relations in the IGI model and refitting it to the large simulated data by the IMM, inter-individual variability assigned to $\mathrm{CL}_{\mathrm{G}}, \mathrm{CL}_{\mathrm{GI}}$, and $\mathrm{k}_{\mathrm{IE}}$ parameters were reduced by $98 \%, 68 \%$, and $98 \%$ respectively as shown in Table I. Since most of the inter-individual variability on $\mathrm{CL}_{\mathrm{G}}$ and $\mathrm{k}_{\mathrm{IE}}$ were explained, but not on $\mathrm{CL}_{\mathrm{GI}}$; the second $\mathrm{IMM}_{\text {parameter }}$ added by the GAM on $\mathrm{CL}_{\mathrm{GI}}$ was incorporated as well, which was the volume of glucose central compartment VOL. That resulted in explaining almost all the remaining inter-individual variability on $\mathrm{CL}_{\mathrm{GI}}$.

\section{DISCUSSION}

Several mathematical models had been developed to improve our understanding and learning about the glucoseinsulin regulatory system, and so that is where modeling 
IMM parameters
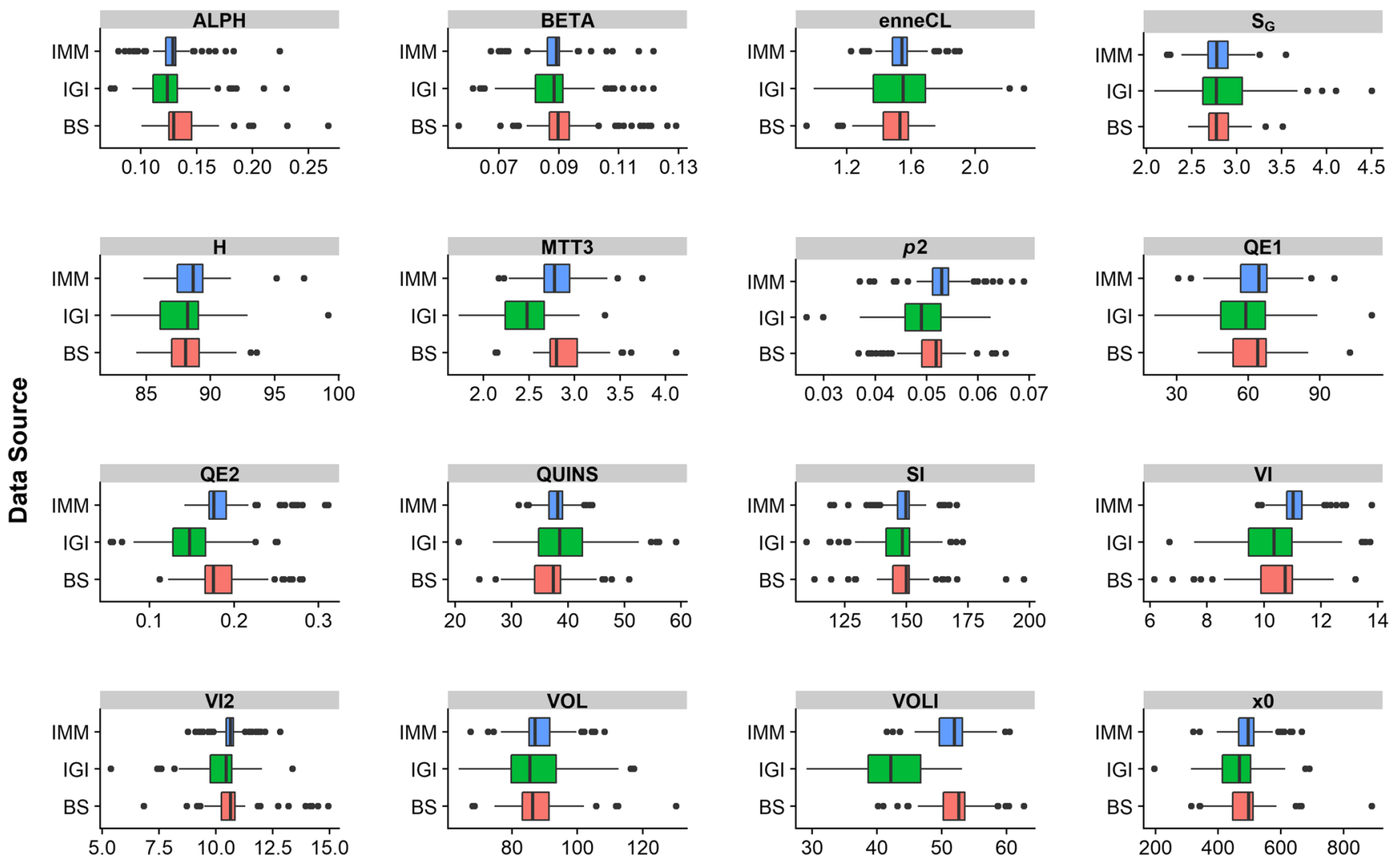

\section{Estimates}

Fig. 7 Boxplots of the estimated distributions of each parameter of the IMM model, grouped by the three different sources of the data: one hundred bootstrap datasets (red), one hundred datasets simulated from the IGI model (green) and one hundred datasets simulated from IMM model (blue). The boxplot length is the interquartile range (the middle half of the distribution) while each whisker represents one quarter of the distribution. BS, bootstrap; IGI, integrated glucose insulin model; IMM, integrated minimal model; ALPH, time constant parameter for insulin provision; BETA, the feedback control of glucose on 2nd phase insulin secretion; enne $\mathrm{CL}$, insulin clearance; $\mathrm{S}_{\mathrm{G}}$, glucose effectiveness; $\mathrm{H}$, glucose basal concentration; $\mathrm{MTT} 3$, insulin I st phase mean transit time; $p 2$, rate parameter for insulin effect; QEI and QE2, inter-compartmental clearance of glucose and insulin respectively; QUINS, insulin basal concentration; SI, insulin sensitivity parameter; $\mathrm{VI}, \mathrm{VI}, \mathrm{VOL}$ and $\mathrm{VOLI}$, volumes of distributions of insulin central compartment, insulin peripheral compartment, glucose central compartment and glucose peripheral compartment respectively; $x 0$,insulin Ist phase concentrations.

has a major impact in the therapeutic area of diabetes. The progress in diabetes modeling was further acknowledged in 2008 with the approval of the first model based computer simulator of human metabolic system for pre-clinical testing of new treatment strategies for type I diabetes (27). In our work, we successfully applied our proposed evaluation

Table I Mapping of Interesting Parameters in the IMM to their Corresponding in the IGI Model

\begin{tabular}{|c|c|c|c|c|c|c|}
\hline IMM parameter & Description & IGI parameter & Description & $\begin{array}{l}\Omega \text { before } \\
\text { inclusion }\end{array}$ & $\begin{array}{l}\Omega \text { After } \\
\text { inclusion }\end{array}$ & $\begin{array}{l}\% \\
\text { change }\end{array}$ \\
\hline $\mathrm{S}_{\mathrm{G}}(\mathrm{d} / \mathrm{min})$ & Glucose effectiveness & $\mathrm{CL}_{\mathrm{G}}(\mathrm{L} / \mathrm{min})$ & $\begin{array}{l}\text { Insulin-independent } \\
\text { glucose clearance }\end{array}$ & 0.142 & 0.003 & $98 \%$ \\
\hline $\mathrm{SI}(\mathrm{L} / \mathrm{min} . \mathrm{pmol})$ & Insulin sensitivity & $\mathrm{CL}_{\mathrm{Gl}}\left(\mathrm{L}^{2} / \mathrm{mU} \cdot \mathrm{min}\right)$ & $\begin{array}{l}\text { Insulin-dependent } \\
\text { glucose clearance }\end{array}$ & 0.276 & 0.086 & $68 \%$ \\
\hline $\mathrm{SI}(\mathrm{L} /$ min.pmol) + VOL (dL) & $\begin{array}{l}\text { Insulin sensitivity + volume of } \\
\text { glucose central compartment }\end{array}$ & $\mathrm{CL}_{\mathrm{Gl}}\left(\mathrm{L}^{2} / \mathrm{m} \cup \cdot \min \right)$ & $\begin{array}{l}\text { Insulin-dependent } \\
\text { glucose clearance }\end{array}$ & 0.276 & 0.006 & $98 \%$ \\
\hline$p 2(/ \mathrm{min})$ & Insulin action & $\mathrm{k}_{\mathrm{IE}}(/ \mathrm{min})$ & Insulin effect rate constant & 0.277 & 0.006 & $98 \%$ \\
\hline
\end{tabular}

$\mathrm{S}_{\mathrm{G}}$, glucose effectiveness; $\mathrm{SI}$, insulin sensitivity; $\mathrm{VOL}$, volumes of distribution of insulin central compartment; $p 2$, rate parameter for insulin action; $\mathrm{CL}_{\mathrm{G}}$, insulinindependent glucose clearance; $\mathrm{CL}_{\mathrm{G}}$, insulin-dependent glucose clearance; $\mathrm{k}_{\mathrm{E}}$, insulin effect rate constant 
procedure on two products of modeling research in diabetes over the past years, the IGI model and the IMM. The results of our work highlighted the pros and cons of the underlying structural assumptions behind each model, and accurately identified existing violations in agreement to previous findings (28).

Both models use a two-compartment disposition model to describe glucose kinetics with elimination from the central compartment only. The parameters of the IMM involved in this were glucose effectiveness $\mathrm{S}_{\mathrm{G}}$, the central compartment volume of distribution VOL, the peripheral compartment volume of distribution VOLI, the intercompartmental clearance QE1, and basal glucose concentrations $H$. All these parameters were well estimated from bootstrap data and the IGI model simulated data except for VOLI which showed underestimation bias with IGI model simulated data. On the other hand, the parameters of the IGI model involved in two compartment description of glucose kinetics were the central compartment volume of distribution $V_{G}$, the peripheral compartment volume of distribution $V_{\mathrm{P}}$, the intercompartmental clearance $Q$ insulin-independent glucose clearance $\mathrm{CL}_{\mathrm{G}}$, insulin-dependent glucose clearance $\mathrm{CL}_{\mathrm{GI}}$ and basal glucose concentrations $\mathrm{G}_{\mathrm{SS}}$. None of these parameters, except $\mathrm{G}_{\mathrm{SS}}$, were well estimated from the IMM simulated data. These results question the simulation performance of IMM, as apparently, the IGI model offers a more informative approach.

For insulin kinetics, the IMM used a two-compartment disposition model with the central compartment volume of distribution VI, the peripheral compartment volume of distribution VI2, the intercompartmental clearance QE2, basal insulin concentrations QUINS and insulin clearance enneCL. Again, all these parameters were well estimated from the IGI model simulated data except for QE2 as expected since insulin kinetics in the IGI model are described with a one compartment disposition model. Interestingly the IGI model simulated data resulted in a more real-life-like estimated VI distribution compared to the IMM itself, and VI2 was precisely estimated from the IGI model simulations which should not be informative for this parameter similar to QE2. The one compartment disposition model of insulin kinetics in the IGI model is described by the central compartment volume of distribution $\mathrm{V}_{\mathrm{I}}$, insulin clearance $\mathrm{CL}_{\mathrm{I}}$, and basal insulin concentrations $\mathrm{I}_{\mathrm{SS}}$. All of these parameters showed differences in the estimated distributions between bootstrap data and the IMM simulated data. Regarding insulin 1 st and 2 nd phase secretions, bootstrap data and the IGI model simulated data showed similar estimated distributions for the IMM parameter BETA, likely different estimated distributions for IMM parameters $\mathrm{x} 0$ and ALPH, and obviously different estimated distributions for IMM parameter MTT3. This could be attributed to the fact that the IGI model assumes no delay in insulin 1 st phase secretion until it reaches the insulin 1 st phase compartment $\mathrm{I}_{\mathrm{FPS}}$ and so its simulations resulted in lower values for mean transit time MTT3 and $\mathrm{x} 0$. In the IGI model, insulin secretions are defined by parameters IFST, IPRG, $\mathrm{k}_{\mathrm{GE} 2}$ and $\mathrm{k}_{\mathrm{IS}}$. Only $\mathrm{k}_{\mathrm{GE} 2}$ was accurately estimated from the IMM simulated data, which is expected as the IMM assume delay in the effect of glucose on insulin 2nd phase secretions through ALPH. Again, the IMM delay in insulin 1st phase secretion led to the higher values of IFST and $\mathrm{k}_{\mathrm{IS}}$. It's important to note that parameters describing insulin secretions in both models were well estimated from bootstrap data and so the two models provided two different ways of capturing the same information. Insulin effect on glucose kinetics in the IMM is described through a remote compartment with different values for the binding rate constant $\mathrm{k}_{2}$ (lumped in $\mathrm{SI}$ ) and the dissociation rate constant $p 2$. The distribution of the insulin sensitivity parameter SI was well estimated from the IGI model simulated data, even though it was not explicitly described in this model, however, the distribution of $p 2$ was not well estimated from the IGI model simulated data, as the IGI model assumed one value for the delay rate constant $\mathrm{k}_{\mathrm{IE}}$. This might also explain the overestimated distribution of the IGI model parameter $\mathrm{k}_{\mathrm{IE}}$ with the IMM simulated data, as here $\mathrm{k}_{\mathrm{IE}}$ should be capturing information about two of the IMM parameters $\mathrm{k}_{2}$ and $p 2$.

The major difference between the two models is their approach of describing endogenous glucose production, this is attributed to the type of data used in the development of each model. Since the IMM adopted the concept of net hepatic glucose balance NHGB and did not explicitly describe a time-varying endogenous glucose production, none of its parameters can reflect on changes in endogenous glucose after system perturbation. While in the IGI model, timevarying endogenous glucose production is explicitly given by an empirical function of basal glucose elimination rate $\mathrm{G}_{\mathrm{SS}} *\left(\mathrm{CL}_{\mathrm{G}}+\mathrm{CL}_{\mathrm{GI}} * \mathrm{I}_{\mathrm{SS}}\right)$, basal glucose concentration $\mathrm{G}_{\mathrm{SS}}$, glucose concentrations in effect compartment $\mathrm{G}_{\mathrm{E} 1}$ and the feedback power term GPRG. All these parameters were well estimated by bootstrap data but not by the IMM simulated data except for $\mathrm{G}_{\mathrm{SS}}$ as shown in Fig. 6. The overestimation of insulin-independent glucose clearance and the underestimation of insulin-dependent glucose clearance by the minimal models was reported previously (4) as a consequence of their implicit way of describing endogenous glucose production. Additionally, the IMM simulated data lacked the needed information for the estimation of the feedback power term GPRG and the delay rate parameter $\mathrm{k}_{\mathrm{GE} 1}$. These results may suggest that the IMM uses a constant direct input of endogenous glucose for healthy subjects following system perturbation, this structure misspecification in the original 
glucose minimal model was early addressed and found to result in a biased parametric description of the whole system (28). Follow-up extensive investigations were performed resulting in the development of the hot glucose twocompartment minimal model (6).

The evaluations using the NLMEPG approach thus revealed some shortcomings of the IMM. Most of these are expected given the structure of the model and the general knowledge of the physiology. However, while these shortcomings were not evident in the VPC, the NLMEPC with the IGI model analysis of the IMM simulated data clearly displayed the difference between these and real data. Also the IGI model simulated data showed shortcomings, although to a lower extent. These results concerning the IGI model can then be considered in the use or update of the IGI model. Thus, primarily the NLMEPG can provide a detailed answer to the question whether the model can generate real-life like data. Secondarily, it may provide further insight into the model deficiencies, when such are present. The information provided on deficiencies may well vary dependent on a number of factors such as the similarity between, and complexity of, the models and the understanding of the underlying system. The data used in this exercise was unlabeled IVGTT data, on purpose selected to be the same type of experimental data as was used to develop the IMM. The IMM model also showed an adequate fit, as judged by the VPC (Fig. 5). The IGI model was developed using labeled IVGTT data and clamp data and the present unlabeled data was only part of the information supporting it. While it may have been an advantage to use for this exercise part of the data from which the model was developed, it is noteworthy that the model appear robust despite much of the data used to develop it was not part of the present data set.

It should be noted that in this exercise we also re-analyzed data with the same model that was used to simulate them. This is not part of the NLMEPG concept, but a procedure sometimes performed to assess whether the estimation method employed is appropriate for the model and data of the analysis in question $(29,30)$.

An important conclusion of our NLMEPG exercise is the fact that the IGI model simulated data contains similar information about the clinical parameters $\mathrm{S}_{\mathrm{G}}$ and $\mathrm{SI}$ as the IMM simulations and bootstrap data, which opens the door for the possibility of deriving these parameters from the IGI model. This fact was further supported by the results from the mapping exercise. Both glucose effectiveness $S_{G}$ in the IMM and insulin-independent glucose clearance $\mathrm{CL}_{\mathrm{G}}$ in the IGI model represented the same glucose elimination mechanism and were derived by insulin-independent rate constants and volumes of glucose central compartment. Thus, the individual estimates of $\mathrm{S}_{\mathrm{G}}$ were the first parameter to be added by the GAM on the individual estimates of $\mathrm{CL}_{\mathrm{G}}$, and the inclusion of the simulated individual estimates of $\mathrm{S}_{\mathrm{G}}$ on population parameter $\mathrm{CL}_{\mathrm{G}}$ in the IGI model resulted in explaining almost all of the unexplained interindividual variability as expected. It must be noticed that even though both parameters carry similar information, only $\mathrm{CL}_{\mathrm{G}}$ measures glucose per se effect on glucose utilization while $\mathrm{S}_{\mathrm{G}}$ is derived from the hybrid rate constant $\mathrm{k}_{5}$ that contains also the inhibitory effect of glucose on endogenous glucose production, and this might be the reason for the overestimation of $\mathrm{CL}_{\mathrm{G}}$ from the IMM simulated data. As insulin sensitivity parameter SI in the IMM is derived from rate constants only (Eq.4), its inclusion alone did not explain all the inter-individual variability of $\mathrm{CL}_{\mathrm{GI}}$, that is derived from both the elimination rate constant and the volume of glucose central compartment. In the stepwise addition to the GAM, the 2nd parameter to be added on $\mathrm{CL}_{\mathrm{GI}}$ was indeed the volume of glucose central compartment VOL in the IMM. Then on including the individual estimates of both SI and VOL as covariates on population parameter $\mathrm{CL}_{\mathrm{GI}}$, most of the interindividual variability was explained. One more interesting parameter derived from the IMM was insulin action $p 2$, this parameter was fully mapped with $\mathrm{k}_{\mathrm{IE}}$ parameter in the IGI model.

From the GAM estimates of our mapping exercise (Eq. 7), we derived (Eq. 8) for the calculation of the individual estimates of $\mathrm{S}_{\mathrm{G}}$ from the IGI model, where $\mathrm{CL}_{\mathrm{G}}$ is the individual estimate of insulin-independent glucose clearance. Similarly, we derived (Eqs. 9-11) for calculating the individual estimates of VOL, SI, and $p 2$ from the IGI model parameters. Noting that the product of SI and VOL as obtained from (Eqs. 9-10) will result in the insulin sensitivity index $\mathrm{S}_{\mathrm{I}}$ provided by the IMM.

$$
\begin{aligned}
& \mathrm{CL}_{\mathrm{G}}=\left(\theta_{\mathrm{CL}_{\mathrm{G}}}+\theta_{\mathrm{EFFECT}} \cdot \mathrm{IMM}_{\mathrm{parameter}}\right) \cdot \exp ^{\eta} \\
& \mathrm{CL}_{\mathrm{G}}=\left(0+0.0829 \cdot \mathrm{S}_{\mathrm{G}}\right) \cdot \exp ^{\eta_{\mathrm{CL}_{\mathrm{G}}}} \\
& \mathrm{S}_{\mathrm{G}}=\mathrm{CL}_{\mathrm{G}} / 0.0829 \\
& \mathrm{VOL}=\left(\mathrm{V}_{\mathrm{G}}-0.9\right) / 0.0899 \\
& \mathrm{SI}=\left(1000 \cdot \mathrm{CL}_{\mathrm{GI}}-0.009 \cdot \mathrm{VOL}\right) /(5094+502 \cdot \mathrm{VOL}) \\
& p 2=\mathrm{k}_{\mathrm{IE}} / 0.938
\end{aligned}
$$

In conclusion, we demonstrated the NMLEPC as a new approach to investigate models' ability to simulate real-lifelike data, and the information captured in each model in comparison to real data, using two glucose homeostasis NLME models. In addition, we proposed a mapping approach for parameters carrying similar information between different NLME models.

\section{ACKNOWLEDGMENTS AND DISCLOSURES}

There is no involvement, financial or otherwise, that might potentially bias this work. 


\section{AUTHOR CONTRIBUTIONS}

M.M.A.I., A.L., M.C.K., and M.O.K. wrote the manuscript. M.M.A.I., A.L., M.C.K., and M.O.K. designed the research. M.M.A.I. performed the research. M.M.A.I., A.L., M.C.K., and M.O.K. analyzed the data.

\section{APPENDIX}

\section{Review of the integrated minimal model (IMM)}

The integrated minimal model IMM shown in Fig. 2, is the integration of two sub-models, glucose minimal model (5) and insulin minimal model (7). Insulin sub-model has twocompartments describing insulin kinetics. In the model, a bolus concentration of insulin $\mathrm{x} 0(\mathrm{pmol} / \mathrm{L})$ is first released in response to the bolus dose of glucose and transit compartments are used to capture the delay in insulin first release, in which the transfer between compartments is govern by the transfer rate parameter $\mathrm{ktr}\left(\mathrm{min}^{-1}\right)$.

$k t r=\frac{n+1}{M T T}$

where $n$ is the number of transit compartments and $M T T(\mathrm{~min})$ is the mean transit time for $\mathrm{x} 0$ to reach insulin central compartment. As the insulin reservoir is replenished, insulin is released proportionally to glucose concentrations in a 2nd phase secretion. The change in 2nd phase insulin secretion is described by the following differential equation:

$\dot{\mathrm{Y}}(t)=-\alpha\left(\mathrm{Y}(t)-\left(\beta\left[g_{1}(t)-g_{b}\right]+k_{I} \cdot I_{b}\right)\right) \quad \mathrm{Y}(0)=k_{I} \cdot I_{b}$

where $\alpha\left(\min ^{-1}\right)$ is the rate parameter describing the delay of $\mathrm{Y}(t)$ towards a new steady state, $\beta\left(\mathrm{min}^{-1} \cdot \mathrm{L}^{-1} \cdot \mathrm{pmol} \cdot \mathrm{dL} \cdot \mathrm{mg}^{-1}\right)$ is glucose sensitivity given by the feedback control of plasma glucose concentrations $g_{1}(t)(\mathrm{mg} / \mathrm{dL})$ above basal glucose concentration $g_{b}(\mathrm{mg} / \mathrm{dL}), k_{I}\left(\mathrm{~min}^{-1}\right)$ is insulin elimination rate parameter and $I_{b}(\mathrm{pmol})$ is basal insulin amount. In the original insulin minimal model, observed plasma glucose concentrations and basal glucose concentrations are used, while in the IMM these concentrations are predicted from glucose submodel. Insulin kinetics are described as:

$$
\begin{aligned}
& \dot{I}_{1}(t)=\mathrm{Y}(\mathrm{t})+I F S+k_{12_{I}} \cdot I_{2}(t)-\left(k_{21 I}+k_{I}\right) \cdot I_{1}(t) \quad I_{1}(0)=I_{b} \\
& \dot{I}_{2}(t)=k_{21 I} \cdot I_{1}(t)-k_{12 I} \cdot I_{2}(t) \quad I_{2}(0)=I_{b}
\end{aligned}
$$

where $I_{1}(t)$ and $I_{2}(t)$ are insulin amounts in the central and peripheral compartments, respectively. IFS ( $\mathrm{pmol} / \mathrm{min})$ is $1^{\text {st }}$ phase insulin secretion rate given as input from the transit compartments, $k_{21 I}$ and $k_{12 I}\left(\mathrm{~min}^{-1}\right)$ are transfer rate parameters. When insulin concentrations in the central compartment is higher than its basal concentrations, it moves to a remote compartment representing receptor pool for insulin binding to peripheral tissues where it produces its effect.

Glucose sub-model is based on the glucose minimal model (5) with a transit compartment input added to describe glucose bolus administration. The model is described by three differential equations, two for glucose kinetics and one for insulin effect on glucose. Insulin effect is exerted by insulin concentrations in the remote compartment representing receptor pool for insulin binding to peripheral tissues.

$\grave{i}=k_{2} \cdot\left(i(t)-i_{b}\right)-k_{3} . \dot{i}(t) \quad i(0)=0$

where $\boldsymbol{l}(\mathrm{pmol} / \mathrm{L})$ is insulin concentrations in the remote compartment, $i(\mathrm{pmol} / \mathrm{L})$ is plasma insulin concentrations, $i_{b}$ $(\mathrm{pmol} / \mathrm{L})$ is basal insulin concentration, $\mathrm{k}_{2}\left(\mathrm{~min}^{-1}\right)$ is the binding rate parameter and $\mathrm{k}_{3}\left(\mathrm{~min}^{-1}\right)$ is the dissociation rate parameter. Observations of plasma insulin and basal insulin concentration are used in the original glucose minimal model, while in the IMM, predicted insulin concentrations from the central compartment of insulin sub-model are used instead. Glucose kinetics are described by two-compartment disposition model, where rate of change of glucose amounts in central compartment $G_{1}^{\prime}(t)$ is described by rate of hepatic glucose production $R a(t)(\mathrm{mg} / \mathrm{min})$, rate of disappearance by liver uptake $R d l(t)(\mathrm{mg} / \mathrm{min})$, rate of disappearance by peripheral tissue uptake $R d p(t)(\mathrm{mg} / \mathrm{min})$ and the distribution between the central and peripheral compartments. Data from IVGTT without labeled glucose did not allow the explicit description of $R a(t)$ so $R a(t)$ and $R d l(t)$ are lumped together as net hepatic glucose balance $\operatorname{NHGB}(t)$ according to:

$$
\dot{G}_{1}=R a(t)-R d l(t)-R d p(t)+k_{12} \cdot G_{2}(t)-k_{21} \cdot G_{1}(t) \quad G_{1}(0)=G_{b}
$$

$\dot{G}_{1}=\operatorname{NHGB}(t)-R d p(t)+k_{12} \cdot G_{2}(\mathrm{t})-k_{21} \cdot G_{1}(\mathrm{t}) \quad G_{1}(0)=G_{b}$

$\operatorname{NHGB}(t)=R a(t)-R d l(t)=B 0-\left(k_{5}+k_{6} \cdot i(t)\right) \cdot G_{1}(t) \quad \operatorname{NHGB}(0)=k_{1} \cdot G_{b}$

$R d p(t)=\left(k_{1}+k_{4} \cdot i(t)\right) \cdot G_{1}(t) \quad R d p(0)=k_{1} \cdot G_{b}$

where $G_{1}(t)$ and $G_{2}(t)$ are glucose amounts in the central compartment and peripheral compartment respectively. $G_{b}(\mathrm{mg})$ is basal glucose amount. $B 0\left(\mathrm{mg} \cdot \mathrm{min}^{-1}\right)$ is the extrapolated NHGB at zero glucose amount, $k_{21}$ and $k_{12}\left(\mathrm{~min}^{-1}\right)$ are transfer rate parameters, $k_{5}\left(\mathrm{~min}^{-1}\right)$ is a rate parameter that describes hepatic glucose uptake as well as the inhibitory effect of glucose on hepatic glucose production, $k_{6}\left(\mathrm{~L}_{\mathrm{pmol}} \mathrm{pmin}^{-1}\right)$ is a rate parameter that describes the effect of insulin concentrations in the remote compartment on glucose to enhance hepatic glucose uptake, as well as the inhibitory effect of insulin on hepatic glucose production, $k_{1}\left(\mathrm{~min}^{-1}\right)$ and $k_{4}$ $\left(\mathrm{L} \cdot \mathrm{pmol}^{-1} \cdot \mathrm{min}^{-1}\right)$ are rate parameters that describe peripheral uptake as a function of glucose amounts in the central 
compartment and insulin concentrations in the remote compartment. By substituting Eqs. (19) and (20) in Eq. (18) and rearranging we obtain:

$\dot{G}_{1}=B 0-\left(k_{5}+k_{1}\right) \cdot G_{1}(\mathrm{t})-\left(k_{6}+k_{4}\right) \cdot i(\mathrm{t}) \cdot G_{1}(t)+k_{12} \cdot G_{2}(\mathrm{t})-k_{21} \cdot G_{1}(t) \quad G_{1}(0)=G_{b}$

with reparameterization as follow (3):

$$
\begin{aligned}
& p 1=k_{1}+k_{5} \\
& p 2=k_{3} \\
& p 3=k_{2} \cdot\left(k_{4}+k_{6}\right) \\
& p 4=\frac{p 3}{p 2} \\
& B 0=p 1 \cdot G_{b} \\
& X(t)=\left(k_{4}+k_{6}\right) \cdot i(t) \quad X(0)=0
\end{aligned}
$$

Leading to the following system of differential equation:

$$
\begin{aligned}
& \dot{G}_{1}=p 1 \cdot G_{b}-\left(p 1+X(\mathrm{t})+k_{21}\right) \cdot G_{1}(\mathrm{t})+k_{12} \cdot G_{2}(\mathrm{t}) \quad G_{1}(0)=G_{b} \\
& \dot{G}_{2}=-k_{12} \cdot G_{2}(\mathrm{t})+k_{21} \cdot G_{1}(\mathrm{t}) \quad G_{2}(0)=k_{21} \cdot G_{b} / k_{12} \\
& \dot{X}=p 4 \cdot p 2 \cdot\left(i(\mathrm{t})-i_{b}\right)-p 2 \cdot X(\mathrm{t}) \quad \mathrm{X}(0)=0
\end{aligned}
$$

\section{Metabolic indices}

Glucose effectiveness $S_{G}\left(\mathrm{dL} \cdot \min ^{-1}\right)$ quantifies the ability of glucose to enhance its own rate of disappearance, at basal insulin concentration (5), and derived as the following equation where $V G_{1}(\mathrm{dL})$ is the volume of glucose central compartment:

$S_{G}=p 1 \cdot V G_{1}=\left(k_{5}+k_{1}\right) \cdot V G_{1}$

Insulin sensitivity $S_{I}\left(\mathrm{~L} \cdot \mathrm{pmol}^{-1} \cdot \mathrm{dL} \cdot \mathrm{min}^{-1}\right)$ quantifies the influence of insulin (at steady state) to change the effect of glucose on its own plasma concentrations (5), $S_{I}$ is derived to be:

$S_{I}=p 4 \cdot V G_{1}=\frac{p 3}{p 2} \cdot V G_{1}=\frac{k_{2}\left(k_{6}+k_{4}\right)}{k_{3}} \cdot V G_{1}$

Basal insulin responsivity to glucose $\Phi_{b}\left(\mathrm{~min}^{-1}\right)$ is the ratio between steady state insulin secretion rate and basal glucose concentration (7).

$\Phi_{b}=\frac{k_{I} \cdot i_{b}}{g_{b}}$

First phase insulin responsivity to glucose $\Phi_{1}$ (dimensionless) is the ratio between the amount of insulin secreted during the first phase and the maximum increase in plasma glucose concentrations $\Delta g(\mathrm{mg} / \mathrm{dL})(7)$.

$\Phi_{1}=\frac{\mathrm{x} 0}{\Delta g}$

Second phase responsivity to glucose $\Phi_{2}\left(\min ^{-1} \cdot \mathrm{L}^{-1} \cdot \mathrm{pmol} \cdot\right.$ $\left.\mathrm{dL} \cdot \mathrm{mg}^{-1}\right)$ is the stimulatory effect of glucose concentrations on 2nd phase insulin secretion (7).

$\Phi_{2}=\beta$

Open Access This article is distributed under the terms of the Creative Commons Attribution 4.0 International License (http://creativecommons.org/licenses/by/4.0/), which permits unrestricted use, distribution, and reproduction in any medium, provided you give appropriate credit to the original author(s) and the source, provide a link to the Creative Commons license, and indicate if changes were made.

\section{REFERENCES}

1. Gobburu JV. Pharmacometrics 2020. J Clin Pharmacol. 2010;50: 151S-7S.

2. Ajmera I, Swat M, Laibe G, Novère NL, Chelliah V. The impact of mathematical modeling on the understanding of diabetes and related complications. CPT Pharmacometrics Sys Pharmacol. 2013;2(7).

3. Bergman RN, Ider YZ, Bowden CR, Cobelli C. Quantitative estimation of insulin sensitivity. Am J Phys. 1979;236:E667-77.

4. Avogaro A, Bristow JD, Bier DM, Cobelli C, Toffolo G. Stablelabel intravenous glucose tolerance test minimal model. Diabetes. 1989;38(8):1048-55.

5. Cobelli C, Caumo A, Omenetto M. Minimal model Sg overestimation and $\mathrm{Si}$ underestimation: improved accuracy by a Bayesian two-compartment model. Am J Physiol Endocrinol Metab. 1999:277:481-8.

6. Toffolo G, Cobelli C. The hot IVGTT two-compartment minimal model: an improved version. Am J Physiol Endocrinol Metab. 2002;284(2)

7. Toffolo G, Campioni M, Basu R, Rizza RA, Cobelli C. A minimal model of insulin secretion and kinetics to assess hepatic insulin extraction. Am J Physiol Endocrinol Metab. 2005;290(1).

8. Denti P, Bertoldo A, Vicini P, Cobelli C. IVGTT glucose minimal model covariate selection by nonlinear mixed-effects approach. Am J Physiol Endocrinol Metab. 2010;298(5):E950-60.

9. Silber HE, Jauslin PM, Frey N, Gieschke R, Simonsson US, Karlsson MO. An integrated model for glucose and insulin regulation in healthy volunteers and type 2 diabetic patients following 
intravenous glucose provocations. J Clin Pharmacol. 2007;47(9): $1159-71$.

10. Largajolli, A., Bertoldo, A.,Cobelli, C., \& Denti, P. An integrated glucose-insulin minimal model for IVGTT, PAGE 22 Abstr 2762; 2013 www.page-meeting. org $/$ ? abstract $=2762$.

11. Girard P, Blaschke TF, Kastrissios H, Sheiner LB. A Markov mixed effect regression model for drug compliance. Stat Med. 1998;17(20):2313-33.

12. Karlsson M, Schoemaker RC, Kemp B, Cohen AF, van Gerven JM, Tuk B, et al. A pharmacodynamic Markov mixed-effect model for the effect of temazepam on sleep. Clin Pharmacol Ther. 2000;68(2):175-88.

13. Yano Y, Beal SL, Sheiner LB. Evaluating pharmacokinetic/ Pharmacodynamic models using the posterior predictive check. J Pharmacokinet Pharmacodyn. 2001;28:171-92.

14. Holford N. The Visual Predictive Check - Superiority to Standard Diagnostic (Rorschach) Plots. PAGE 14 Abstr 738; 2005. www. page-meeting.org $/$ ?abstract $=738$.

15. Karlsson MO, Holford N. A Tutorial on Visual Predictive Checks. PAGE 17 Abstr 1434; 2008. www.page-meeting.org/? abstract $=1434$.

16. Bergstrand M, Hooker AC, Wallin JE, Karlsson MO. Predictioncorrected visual predictive checks for diagnosing nonlinear mixedeffects models. AAPS J. 2011;13:143-51.

17. Jauslin PM, Karlsson MO, Frey N. Identification of the mechanism of action of a Glucokinase activator from Oral glucose tolerance test data in type 2 diabetic patients based on an integrated glucoseinsulin model. J Clin Pharmacol. 2012;52(12):1861-71.

18. Ghadzi SM. Pharmacometrics Modelling in Type 2 Diabetes Mellitus: Implications on Study Design and Diabetes Disease Progression. (Doctoral dissertation). Uppsala: Acta Universitatis Upsaliensis. 2017

19. Ibrahim MMA, Ghadzi SMS, Kjellsson MC, Karlsson MO. Study design selection in early clinical anti-hyperglycemic drug development: a simulation study of glucose tolerance tests. CPT Pharmacometrics Sys Pharmacol. 2018;7(7):432-41.

20. Silber HE, Nyberg J, Hooker AC, Karlsson MO. Optimization of the intravenous glucose tolerance test in T2DM patients using optimal experimental design. J Pharmacokinet Pharmacodyn. 2009;36(3):281-95.
21. Vicini P, Caumo A, Cobelli C. The hot IVGTT two-compartment minimal model: indexes of glucose effectiveness and insulin sensitivity. Am J Phys. 1997;273:E1024-32.

22. Vicini P, Zachwieja JJ, Yarasheski KE, Bier DM, Caumo A, Cobelli C. Glucose production during an IVGTT by deconvolution: validation with the tracer-to-tracee clamp technique. Am J Phys. 1999;276:E285-94.

23. Hastie TJ, Tibshirani RJ. Generalized additive models: Chapman \& Hall/CRC . ISBN; 1990. isbn:978-0-412-34390-2.

24. Lindbom L, Pihlgren P, Jonsson EN. PsN-toolkit - a collection of computer intensive statistical methods for non-linear mixed effect modeling using NONMEM. Comput Methods Prog Biomed. 2005;79(3):241-57.

25. Beal S, Sheiner LB, Boeckmann A, Bauer RJ. NONMEM User's guides. USA: Icon Development Solutions; 2009. p. 1989-2009.

26. Team RC. R: a language and environment for statistical computing. Vienna, Austria: 2014. Available from: https://www.R-project.org.

27. Patek SD, Bequette BW, Breton M, Buckingham BA, Dassau E, Doyle FJ, et al. In silico preclinical trials: methodology and engineering guide to closed-loop control in type 1 diabetes mellitus. J Diabetes Sci Technol. 2009;3(2):269-82.

28. Cobelli C, Pacini G, Toffolo G, Saccà L. Estimation of insulin sensitivity and glucose clearance from minimal model: new insights from labeled IVGTT. Am J Phys. 1986;250(5):E591-8.

29. Karlsson MO, Jonsson EN, Wiltse CG, Wade JR. Assumption testing in population pharmacokinetic models: illustrated with an analysis of Moxonidine data from congestive heart failure patients. $\mathrm{J}$ Pharmacokinet Biopharm. 1998;26:207-46.

30. Bizzotto R, Zamuner S, Mezzalana E, Nicolao GD, Gomeni R, Hooker AC, et al. Multinomial logistic functions in Markov chain models of sleep architecture: internal and external validation and covariate analysis. AAPS J. 201 1;13:445-63.

Publisher's Note Springer Nature remains neutral with regard to jurisdictional claims in published maps and institutional affiliations. 